\title{
Short Term Outcomes of Laparoscopic Mini-Gastric Bypass/ One Anastomosis Gastric Bypass. A Single Center Experience
}

Islam S. Radwan, Mohamed A. Hablus, Soliman M. Soliman and Hosam B. Barakat*

*Correspondence: elbohotyhossam2018@gmail.com

CrossMark

\& Click for updates

Gastrointestinal and Laparoscopic Surgery Unit, General Surgery Department, Faculty of Medicine, Tanta University, Egypt.

\begin{abstract}
Background: Laparoscopic One-anastomosis gastric bypass (LOAGB) has proved to be safe and effective, with short learning curve and low morbidity rate.

Methods: This was a prospective study done from September 2017 to August 2019. Forty morbidly obese patients (27 female, 13 male) were included and all were subjected to Laparoscopic One-anastomosis gastric bypass. This study discussed the patients' demographics, operative technique, operative records, post-operative outcomes concerning excess weight loss, comorbidities resolution and morbidity rates. Follow up was done at 1, 3, 6, 12 and 24 months postoperative.

Results: Mean patients' age was $38.5 \pm 9.45$ years. Mean patients' BMI was $53.59 \pm 6.25 \mathrm{~kg} / \mathrm{m} 2$. All procedures were completed laparoscopically. Mean operative time was 94.125 333.20 min (range, 65-230 min). The mean length of hospital stay was $2.025 \pm 1.32$ day. Mean percentage of excess weight loss achieved was $21.03 \pm 3.32 \%, 38.02 \pm 5.15 \%, 58.095 \pm 5.83 \%, 70.98 \pm 7.29 \%$, and $78.64 \pm 10.3 \%$ at $1,3,6,12$ and 24 month respectively. Most of co-morbidites were improved or resolved within the first postoperstive year. There was no perioperative mortality whereas total morbidity occurred in 9 patients (22.5\%).
\end{abstract}

Conclusion: LOAGB is feasible, safe with short hospital stay and accepted morbidity rate. Short term outcomes concerning promotion of weight loss and remission/improvement of obesity associated comorbidities are promising.

Keywords: Bariatric surgery, Morbid obesity, One anastomosis gastric bypass, Short term, Weight loss

\section{Introduction}

The growing concern with obesity all over the world is attributed not only to dramatic prevalence but also to great association with several comorbidities as type 2 diabetes mellitus, hypertension, obstructive sleep apnea, dyslipidemia, gastroesophageal reflux disease, and certain types of cancer [1]. Multiple studies have demonstrated that bariatric surgery for morbid obese populations produces successful and sustained weight loss, and result in reduction of obesity-related comorbidities and improving the quality of life compared with non-surgical means [2]. The One-anastomosis gastric bypass (OAGB), also known as mini-gastric bypass (MGB) or omega-loop gastric bypass, was first described by Rutledge as a modification of the Mason's loop gastric bypass [3]. It was proposed as a simple and efficient bariatric procedure with weight loss results comparable to laparoscopic Roux-en-Y gastric bypass (LRYGB) but with shorter operative times and lower risk of leaks from one less anastomotic site $[4,5]$. The aim of this study was to report our experience in LOAGB and evaluating short term results regarding safety and efficacy.

\section{Patients and Methods}

This was a prospective study carried on 40 morbidly obese patients ( 27 female, 13 male) at the Gastrointestinal and Laparoscopic Surgery Unit, Department of General Surgery, Tanta University Hospital, during the period from September 2017 to August 2019.

\section{Inclusion criteria}

Age between 20 and 60 years and fulfillment of the criteria of National Institutes of Health Development Panel (BMI > $35 \mathrm{~kg} /$ $\mathrm{m} 2$ with one or more obesity related comorbidities; or BMI $\geq 40$ 
Barakat et al., Chronicles of Surgery 2021,

http://www.hoajonline.com/journals/pdf/2053-7212-9-1.pdf

doi: 10.7243/2053-7212-9-1

$\mathrm{kg} / \mathrm{m} 2$ regardless the presence or absence of comorbidities).

\section{Exclusion criteria}

Were patients younger than 20 and older than 60 years, history of alcohol or drug dependence, patients classified as American Society of Anesthesiologists (ASA) grade IV, Patients with symptomatic reflux, patients with previous bariatric surgery, patients with previous upper abdominal surgery and all patients with psychiatric impairment. All of our candidates were fully informed about the expected outcomes, benefits and possible complications of this procedure and signed a written informed consent. The study was approved by institutional ethical committee.

\section{Preoperative Work-Up}

Preoperative evaluation of our candidates by a multidisciplinary team (surgeons, nutritionist, psychologist, cardiologist, and anesthesiologist) was essential. Routine laboratory investigations in addition to complete lipid profile and hormonal assay to exclude cases of secondary obesity (Cushing's syndrome and hypothyroidism) were done for all patients. Radiological studies including abdominal ultrasound, echocardiography and chest $\mathrm{x}$-ray were done for all patients whereas upper gastrointestinal endoscopy was applied only for selected cases. Prophylactic dose of low molecular-weight heparin was used for high-risk patients to guard against deep venous thrombosis (DVT). All patients were put on a low calorie high protein diet regimen for a minimum of 7 days preoperatively.

\section{Statistical analysis}

Results were tabulated and statistically analyzed using MICROSOFT EXCEL 2017 and SPSS V.25 program for MICROSOFT WINDOWS 10.

\section{Surgical Technique}

All procedures were done by the same surgeons (authors). Following general anesthesia, the patient was positioned in the supine leg splitting position, the surgeon stood on the right side, the camera man stood in between the patient's leg and the assistant stood on the patient left. Five trocars are usually used, three $(12 \mathrm{~mm})$ and two $(5 \mathrm{~mm})$, Figure 1 . The first $12 \mathrm{~mm}$ optical trocar was placed $15-18 \mathrm{~cm}$ caudal to the xiphoid process 1 inch to the left of midline for the $30^{\circ}$ optical system, the second $12 \mathrm{~mm}$ port is inserted at the same level of the camera port 1 inch to the right of the midline (surgeon's right hand), the third $12 \mathrm{~mm}$ port inserted $1 \mathrm{~cm}$ below the right costal margin just lateral to midclavicular line (surgeon's left hand). The first $5 \mathrm{~mm}$ port (assistant) is inserted $5 \mathrm{~cm}$ below the left costal margin in the midclavicular line. The second 5 $\mathrm{mm}$ port is inserted in the midline just below xiphisternum (for liver retraction). After abdominal exploration on initial laparoscopy, we begin the operation by the creation of the lesser omental window by the harmonic scalpel (Ethicon Endo-Surgery, Cincinnati, Ohio, USA) at the level of crow's

foot, Figure 2, then the stomach is divided horizontally at the level of incisura using $40 \mathrm{~mm}$ of a $60 \mathrm{~mm}$ blue load linear stapler (Echelon Flex Endopath; Ethicon Endo-Surgery Inc., Johnson and Johnson, Cincinnati, Ohio, USA) introduced through the created window. Once the $1^{\text {st }}$ stapler fired, this creates the new base of the gastric pouch and will be the site of the gastrojejunostom, Figure 3. The second stapler is applied parallel to the gastric lesser curvature, Figure 4. Further firing is done vertically upward parallel to lesser curvature and along orogastric calibration tube 36 French till the angle of Hiss where an opening in the esophagogastric ligament is created to expose the lateral aspect of the left crus of diaphragm, Figure 5 , to be followed by the last stapler. It is essential not to leave any communication between the gastric pouch and the gastric remnant and to inspect the staple lines carefully for hemostasis. The greater omentum and the transverse colon are retracted cephalad to identify the Treitz ligament then a loop of jejunum $180 \mathrm{~cm}$ from duodenojejunal flexure was
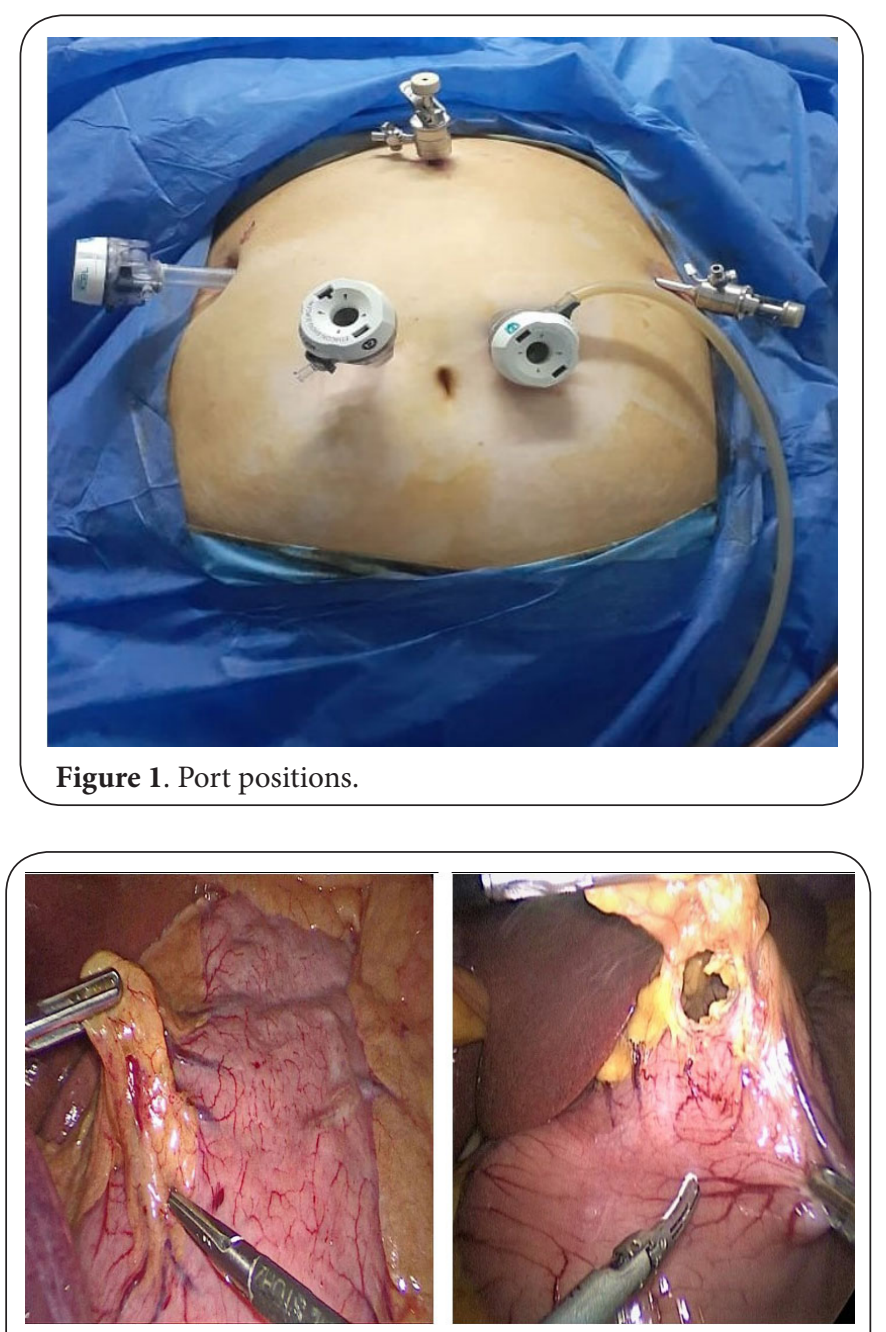

Figure 2. a: Site of beginning of dissection on lesser curvature. b: Creation of of the lesser omental window at the level of crow's foot. 

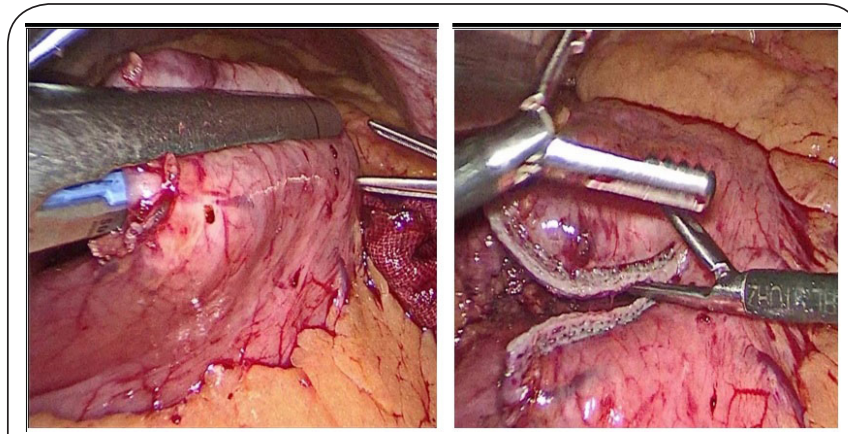

Figure 3 a: Application of the 1st stapler through the created window perpendicular to the lesser curvature.

b: the 1st stapler is fired creating the base of the gastric pouch.

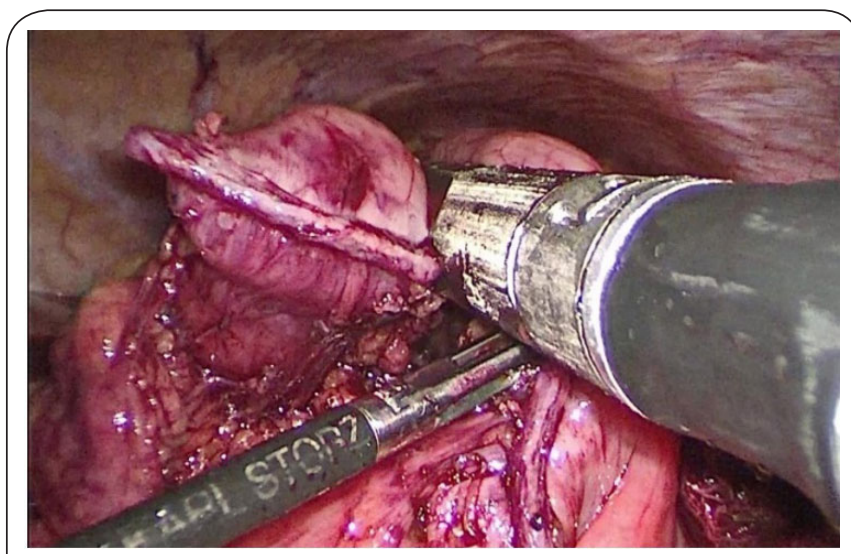

Figure 4. The 2nd stapler applied parallel to the gastric lesser curvature.

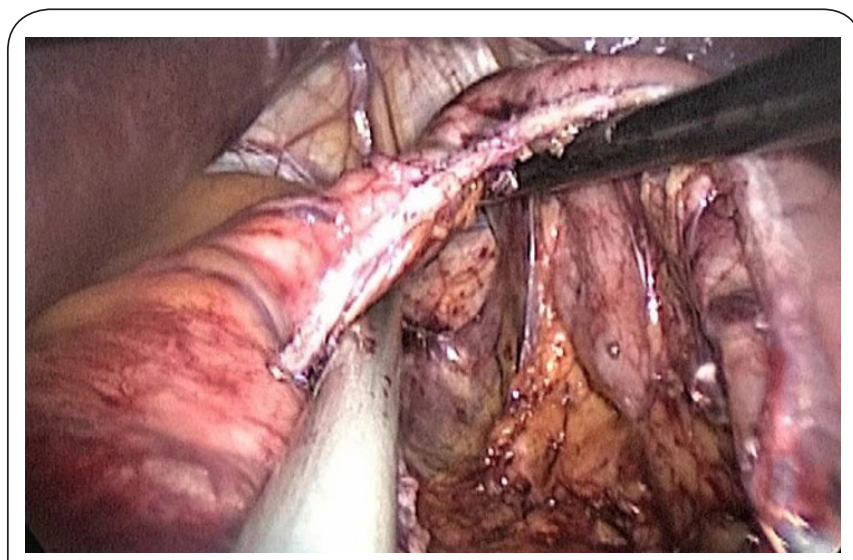

Figure 5. Exposure of left crus of diaphragm before application of the last stapler.

then brought up to the level of gastric pouch in an ante colic manner and side-to-side gastrojejunostomy anastomosis was created using $40-50 \mathrm{~mm}$ of a $60 \mathrm{~mm}$ blue load linear stapler and the stapler entery site was then closed in one layer by running an absorbable suture (Vicryl 3-0 suture) Figure 6, to be followed by methylene blue leakage test. The afferent limb

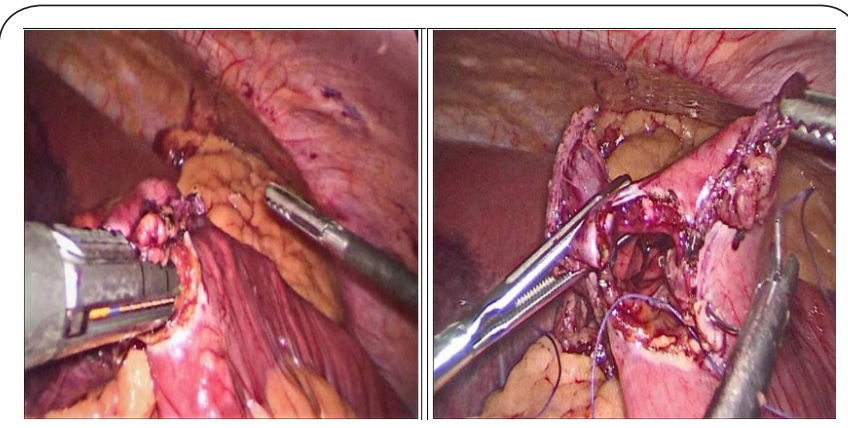

Figure 6. a: Creation of gastrojejunostomy.

b: The stapler entery site is closed by running an absorbable suture (Vicryl 3-0 suture).

then fixed to the gastric pouch using interrupted sutures. Finally, an intra-abdominal drain was routinely placed beside the anastomosis site in all cases.

\section{Results}

The study population included 40 patients, involving 27 (67.5\%) females and 13 (32.5\%) male. The patients' demographic characteristics illustrated in Table 1. Overall, 31 patients attended 24 months of follow-up. A wide range of obesity related comorbidities were found in our study; the commonest was osteoarthritis in 24 patients $(60 \%)$, dyslipidemia in 16 patients $(40 \%)$, hypertension in 13 patients (32.5\%), Diabetes mellitus (DM) in 10 patients (25\%), gallbladder stones in 3 patients (7.5\%). ischemic heart disease in one patient (2.5\%), obstructive sleep apnea (OSA) in 4 patients (10\%), urinary problems (stress incontinence) in 2 patients (5\%) and varicose veins in 3 patients (7.5\%).

\section{Operative records and hospital stay}

All procedures were completed laparoscopically with no conversions. The median jejunal limb length was $180 \mathrm{~cm}$ (range 150-200 cm). Concomitant cholecystectomy through the same ports was done for 3 cases $(7.5 \%)$ due to presence of G.B stone. Regarding Intraoperative mishaps, superficial liver laceration in 6 patients and were managed laparoscopically by electro-cautery, also, the operation was aborted in one female patient due to huge fatty liver. The mean operative time was $94.125 \pm 33.20 \mathrm{~min}$ (range, $65-230 \mathrm{~min}$ ). The mean length of hospital stay was $2.025 \pm 1.32$ day (range: 1-7 day).

Table 1: Patients' demographic data.

\begin{tabular}{lll}
\hline & Rang & Mean \pm SD \\
\hline Age & $20-55$ & $38.5 \pm 9.45$ \\
Weight/kg & $103-209$ & $151.85 \pm 23.37$ \\
Height/cm & $157-187$ & $168.35 \pm 7.61$ \\
BMI $(\mathbf{k g} / \mathbf{m} 2)$ & $38.29-71.12$ & $53.59 \pm 6.25$ \\
Excess weight/kg & $36-128$ & $80.88 \pm 20.64$ \\
\hline
\end{tabular}


Barakat et al., Chronicles of Surgery 2021,

\section{Morbidity and mortality}

No mortality occurred in this study. Early postoperative complications (within the first month postoperative) occurred in 4 patients (10\%). Pulmonary embolism recorded in one patient necessitating ICU admission and improved. Two patients (5\%) developed early postoperative bleeding with drainage volume (>100 cc/hour), conservative measures was beneficial in one case while re-laparoscopy was essential in the other case, where the source was the stable line of the excluded stomach and was managed by under running sutures. One patient $(2.5 \%)$ had wound infection and was managed by antibiotics and daily dressing. There was no anastomotic leakage. Late complications were encountered in 5 cases (12.5\%) and listed in Table 2. There was no bile reflux, marginal ulcers or weight regain.

Table 2: Late complications.

\begin{tabular}{lll}
\hline Late complications & Number & $\mathbf{( \% )}$ \\
\hline Iron deficiency anaemia & 2 & $5 \%$ \\
Symptomatic cholethiasis & 2 & $5 \%$ \\
Insufficient weight loss & 1 & $2.5 \%$ \\
Total & 5 & $12.5 \%$ \\
\hline
\end{tabular}

\section{Weight loss results}

Mean EWL\% was $21.03 \pm 3.32 \%, 38.02 \pm 5.15 \%, 58.095 \pm 5.83 \%$, $70.98 \pm 7.29 \%$, and $78.64 \pm 10.3 \%$ at $1,3,6,12$ and 24 month respectively. All patients lost weight during the first year, however, one patient lost weight but not efficiently (EWL\% less than $50 \%$ at one year).

\section{Resolution of comorbidities}

After the first postoperative year, Most of the co-morbidities improved or resolved, Table 3.

\section{Discussion}

The purpose of this study was to report short term outcomes of OAGB in management of morbid obese patients. In this study, the main operative time was $94.125 \pm 33.20$ min which was similar to that reported by Wang et al, (95 $\pm 41.5 \mathrm{~min})$ [6], and Carbajo et al. (93 min) [7], however it was longer than that reported by Jammu and Sharma (57.5 minute) [8], and kuller et al (reported $52 \mathrm{~min}$ ) [9]. On the other hand, it was shorter than that reported by Alkhalifah et al (reported $124.6 \pm 38.8 \mathrm{~min})$ [10] and Fetouh et al (126.83 \pm 29.43$) \mathrm{min}$
[11]. This variation of operative time between different studies may be attributed to different surgeons' experience and concomitant operations. In this study, the main length of hospital stay was $2.025 \pm 1.32$ day which was comparable to other published studies [7,9]. In this study, the excess weight loss was $58.09 \%, 70.98 \%$, and $78.64 \%$ after 6, 12 and 24 month respectively. This was in agreement with the study carried out by Sczepaniak et al. [12] who reported the mean EWL $\%$ of $54,7 \%$ and $69.4 \%$ after 6 and 12 month follow up respectively of their patients subjected to LOAGB. Also, Abd Al-Motalib et al., [13] reported similar results regarding EWL\% of $72 \%$.at 12 month follow up. Kular et al, [9], reported a higher mean EWL\% which was $85 \%$ and $91 \%$ after 12 and 24 month. The variation in EWL\% between different studies may be attributed to different bougie diameter applied for creation of gastric pouch, different jejunal limb length or may be related to different patients' culture. In this study, most of the comorbidities improved or resolved after the first 12 months postoperatively. Resolution/improvement of hypertension was $84.6 \%$. Our results are in agreement with that reported by Jammu and Sharma [8] which was $85.4 \%$. In this study, ten patients had type 2 DM (6 patient were on a single oral hypoglycemic therapy while, 4 patient were on subcutaneous insulin therapy). Resolution/improvement of type 2 DM at 1-year follow-up was $90 \%$ (total resolutions in 8 patients and improvement in one patient). Complete remission of diabetes was significantly higher in patients who were on preoperative oral hypoglycemic drugs than in those who were on subcutaneous insulin therapy, $100 \%$ versus $50 \%$ respectively $(\mathrm{p}<0.01)$. Taha et al. [14] reported DM remission rate of $92.2 \%, 95.2 \%$ and $72.4 \%$ in patients who were preoperatively on a single, two and three oral hypoglycemic drugs therapy respectively. Also, they reported DM remission of $52 \%$ in their patients with preoperative insulin therapy. In our study, resolution/improvement of dyslipidemia, osteoarthritis, sleep apnea and urinary incontinence were $93.75 \%, 87.5 \%, 100 \%$ and $100 \%$ respectively which were comparable to other published studies $[8,9,15]$. In our study, all procedures were accomplished by laparoscopically with no need for conversion to open surgery. Perioperative mortality was zero (0\%), early postoperative complication were seen in 4 patients (10\%), one patient was diagnosed as pulmonary embolism and were treated with anticoagulant therapy in the ICU, two patients (5\%) developed intraabdominal bleeding, one of them managed conservatively while relaparoscopy

Table 3: Post-operative comorbidities change after 1st year postoperative.

\begin{tabular}{lllllll}
\hline Co-morbidity & DM & Hypertension & OSA & Osteoarthritis & Dyslipidemia & Urinary \\
\hline Remission & 8 & 6 & 2 & 8 & 11 & - \\
improvement & 1 & 5 & 2 & 13 & 4 & 2 \\
No change & 1 & 2 & & 3 & 1 & \\
Total & 10 & 13 & 4 & 24 & 16 & 2 \\
\hline
\end{tabular}

DM: Diabetes mellitus, OSA: Obstructive sleep apnea 
Barakat et al., Chronicles of Surgery 2021,

was essential in the other, one patient had wound infection . There was no reported case of leakage or Jejunal perforation. This was in agreement with Abd Al-Motalib et al [13], reported early postoperative complications in $4 \%$ of their patients where $2 \%$ had abdominal and GIT bleeding and $2 \%$ had respiratory distress with no cases of leakage or jejunal perforation. Piazza et al. [16], in a series of 197 cases reported one case mortality and early postoperative complications in eight (4\%) patients and included pulmonary embolism in 2 patients and melena in six patients, and all patients were treated conservatively. Wang et al. [6], in a series of 423 cases reported 2 cases mortality, early postoperative complications in 25 patients (6\%) and included 9 patients with anastomotic leakage, 7 patients with anastomosis bleeding, 5 patients with wound infection, 1 patient with gastric stasis, 1 patient with mechanical ileus, 1 patient with bleeding duodenal ulcer and 1 patient with suturing of the nasogastric tube. In our study, late postoperative complications developed in 5 patients (12.5\%). Two patients developed sever iron deficiency anaemia that was evident within 6 month post operatively and required parenteral iron supplementation, two patient developed symptomatic cholethiasis and were subjected to laparoscopic cholecystectomy, one patient experienced insufficient weight loss after the first year postoperatively with EWL $\%>50 \%$. No cases of malnutrition were detected in this study. Rutledge and Walash [17], in a series of 2410 patients reported late complications in $11.7 \%$, and included dyspepsia and ulcer in 135 (5.6\%) patients, iron-deficiency anemia in $110(5 \%)$ patients, and malnutrition due to excessive weight loss in 31 (1.1\%) patients with no reported cases of reflux. Abd Al-Motalib et al [13], reported late postoperative complications in 6 patients $(6 \%)$ and included iron-deficiency anemia in 4 patients and Interactable reflux in 2 patients. Wang et al, [6], reported iron deficiency Anemia as the most frequent late complication in 41 patients (9.7\%); and marginal ulcers in 34 patients (8.0\%). There are several controversial aspects surrounding LOAGB that have discouraged a wider adoption of this procedure. One of these controversies is the risk postoperative symptomatic biliary reflux [18]. In our study, no cases of symptomatic biliary reflux were detected during follow up which was in agreement with some published studies $[7,17,19,20]$. Some factors that may explain why LOAGB doesn't increase biliary reflux include: Firstly, the pouch, which is lengthier and narrower than that of the classic gastric bypass, is designed to understate the reflux of enteric secretion through the anastomosis. Secondly, the anastomosis itself, which is vertical or slightly oblique in the posterior wall of the pouch, favors the gastric emptying and potentially avoids significant reflux. Furthermore, the distance from the Treitz angle, composing a long biliopancreatic limb (around $200 \mathrm{~cm}$ ) permits the resorption of large amounts of the biliary secretion; thus, the enteric juice that arrives at the anastomosis site is not so concentrated as the one which usually arrived at the Billroth II gastrectomy design $[3,17,18]$. In this study, we found that LMGB a great effect on weight reduction,
BMI and resolution /improvement on most of co-morbidites. The major limitations of this study are the limited populations sample who subjected to LOAGB and the short postoperative follow-up period; Further long term studies are recommended to provide more evidence regarding the long-term outcomes.

\section{Conclusion}

MGB/OAGB is a simple, safe, highly efficient bariatric procedure, easy to perform with short learning curve. It provides not only excellent weight loss results, but also, resolution or improvement of obesity associated comorbidities during short term follow up. The low morbidity and mortality rates associated with LOAGB making it an ideal weight loss operation. Further longterm studies are recommended.

\section{List of abbreviations}

DM: Diabetes mellitus

LOAGB: Laparoscopic One-anastomosis gastric bypass

LRYGB: laparoscopic RouxenY gastric bypass

MGB: Mini-gastric bypass

DVT: Deep venous thrombosis

\section{Competing interests}

The authors declare that they have no competing interests.

Authors' contributions

\begin{tabular}{|l|c|c|c|c|}
\hline Authors' contributions & ISR & MAH & SMS & HBB \\
\hline Research concept and design & -- & $\checkmark$ & $\checkmark$ & $\checkmark$ \\
\hline Collection and/or assembly of data & $\checkmark$ & -- & -- & $\checkmark$ \\
\hline Data analysis and interpretation & $\checkmark$ & $\checkmark$ & $\checkmark$ & $\checkmark$ \\
\hline Writing the article & $\checkmark$ & $\checkmark$ & -- & $\checkmark$ \\
\hline Critical revision of the article & $\checkmark$ & $\checkmark$ & $\checkmark$ & $\checkmark$ \\
\hline Final approval of article & $\checkmark$ & $\checkmark$ & $\checkmark$ & $\checkmark$ \\
\hline Statistical analysis & -- & -- & -- & -- \\
\hline
\end{tabular}

Publication history

Editor: Sawsan Abuhamdah, University of Jordan, Jordan.

Received: 21-June-2021 Final Revised: 28-Aug-2021

Accepted: 10-Sept-2021 Published: 29-Sept-2021

\section{References}

1. Collins J, Meng C, Eng A. Psychological impact of severe obesity. Curr Obes Rep. 2016; 5(4):1-6.

2. Angrisani $L$, Santonicola $A$, lovino $P$, et al. Bariatric surgery and endoluminal procedures: IFSO Worldwide Survey 2014. Obes Surg. 2017; 27(9): 2279-89.

3. Rutledge R. The Mini-Gastric Bypass: Experience with the First 1,274 Cas es. Obes Surg. 2001;11: 276-280

4. Chaim EA, Ramos AC, Cazzo E. Mini-gastric bypass: description of the technique and preliminary results. Arq Bras Cir Dig (São Paulo). 2017; 30:264-6

5. Mahawar KK, Kumar P, Carr WR, et al. Current status of mini-gastric bypass. J Minim Access Surg. 2016; 12 (4): 305-10.

6. Wang W, Wei P, Lee Y, Huang M, Chiu C, Lee W. Short-term Results of Laparoscopic Mini-Gastric Bypass. Obes Surg. 2005; 15, 648-654.

7. Carbajo M., M. Garcia-Caballero, M. Toledano, D. Osorio, C. Garcıa-Lanza, 
Barakat et al., Chronicles of Surgery 2021,

http://www.hoajonline.com/journals/pdf/2053-7212-9-1.pdf

and J. A. Carmona, "One-anastomosis gastric bypass by laparoscopy: results of the first 209 patients, " Obes Surg. 2005; 15(3):.398-404.

8. Jammu GS, Sharma R. A 7-year clinical audit of 1107 cases comparing sleeve gastrectomy, Roux-En-Y gastric bypass, and mini-gastric bypass, to determine an effective and safe bariatric and metabolic procedure. Obes Surg. 2016; 26:926-932.

9. Kular \& N. Manchanda \& R. Rutledge. A 6-Year Experience with 1,054 Mini-Gastric Bypasses-First Study from Indian Subcontinent K. S. Obes Surg. 2014; 24:1430-1435 DOI 10.1007/s11695-014-1220-3.

10. Alkhalifah N, Lee WJ, Hai TC, et al. 15-year experience of laparoscopic single anastomosis (mini-)gastric bypass: comparison with other bariatric procedures. Surg Endosc. 2018;32(7):3024-31.

11. Fetouh A, Saber S, Elmahdy T, Swelam A, Elgarf S, Abdallah H, Moussa G. Outcomes of laparoscopic one-anastomosis gastric bypass in treatment of morbid obesity: a retrospective analysis. Egyptian J Surgery 38:713-721.

12. Sczepaniak JP, Owens ML, Shukla H, Perlegos J, Garner W Comparability of weight loss reporting after gastric bypass and sleeve gastrectomy using BOLD data 2008-2011. Obes Surg. 2015; 25:788-795.

13. Abd Al-Motalib HA , Taha OY, Sleem AA Mohamed AM. Laparoscopic MiniGastric Bypass in Management of Morbid Obesity A Prospective Study. The Egyptian Journal of Hospital Medicine. 2020;79: 384-391.

14. Taha O, Abdelaal M, Abozeid M et al. Outcomes of One Anastomosis Gastric Bypass in 472 Diabetic Patients. Obes Surg. 2017; 27: 2802-2810.

15. Carbajo MA, Luque-de-Leon E, Jiménez JM et al.: Laparoscopic one-anastomosis gastric bypass: technique, results, and long-term follow-up in 1200 patients. Obes Surg., 2017; 27: 1153-67.

16. Piazza L, Ferrara F, Leanza S, Coco D, Sarvà S, Bellia A, et al. Laparoscopic mini-gastric bypass: short-term single-institute experience. Updates Surg 2012; 63:239-242

17. Rutledge R, Walsh TR. Continued excellent results with the minigastric bypass: six-year study in 2,410 patients. Obes Surg. 2005;15(9):1304-8.

18. Mahawar KK, Carr WR, Balupuri S, Small P. Controversy surrounding 'mini' gastric bypass. Obes Surg. 2014; 24(2):324-33.

19. Kim Z. and K. Y. Hur, "Laparoscopic minigastric bypass for type 2 diabetes: the preliminary report," World Journal of Surgery 2011; 35(3): 631-636.

20. Noun R., J. Skaff, E. Riachi, R. Daher, N. A. Antoun, and M. Nasr, “One thousand consecutive mini-gastric bypass: short and longterm outcome", Obes Surg. 2012; 22 (5): 697- 70.

\section{Citation:}

Radwan IS, Hablus MA, Soliman SM and Barakat HB. Short Term Outcomes of Laparoscopic Mini-Gastric Bypass/One Anastomosis Gastric Bypass. A Single Center Experience. Chron Surg. 2021; 9:1. http://dx.doi.org/10.7243/2053-7212-9-1 